\title{
Beer Potomania Masquerading as Adrenal Insufficiency
}

L Hopkins, V Stokes, S Chatterjee

Endocrinology Department, Stoke Mandeville Hospital, Aylesbury, Buckinghamshire, UK HP21 8AL

\section{Abstract}

\section{Investigations}

Follow Up

Other baseline pituitary function tests were

organised to investigate this patient further:

- MRI head revealed a structurally normal

pituitary gland.

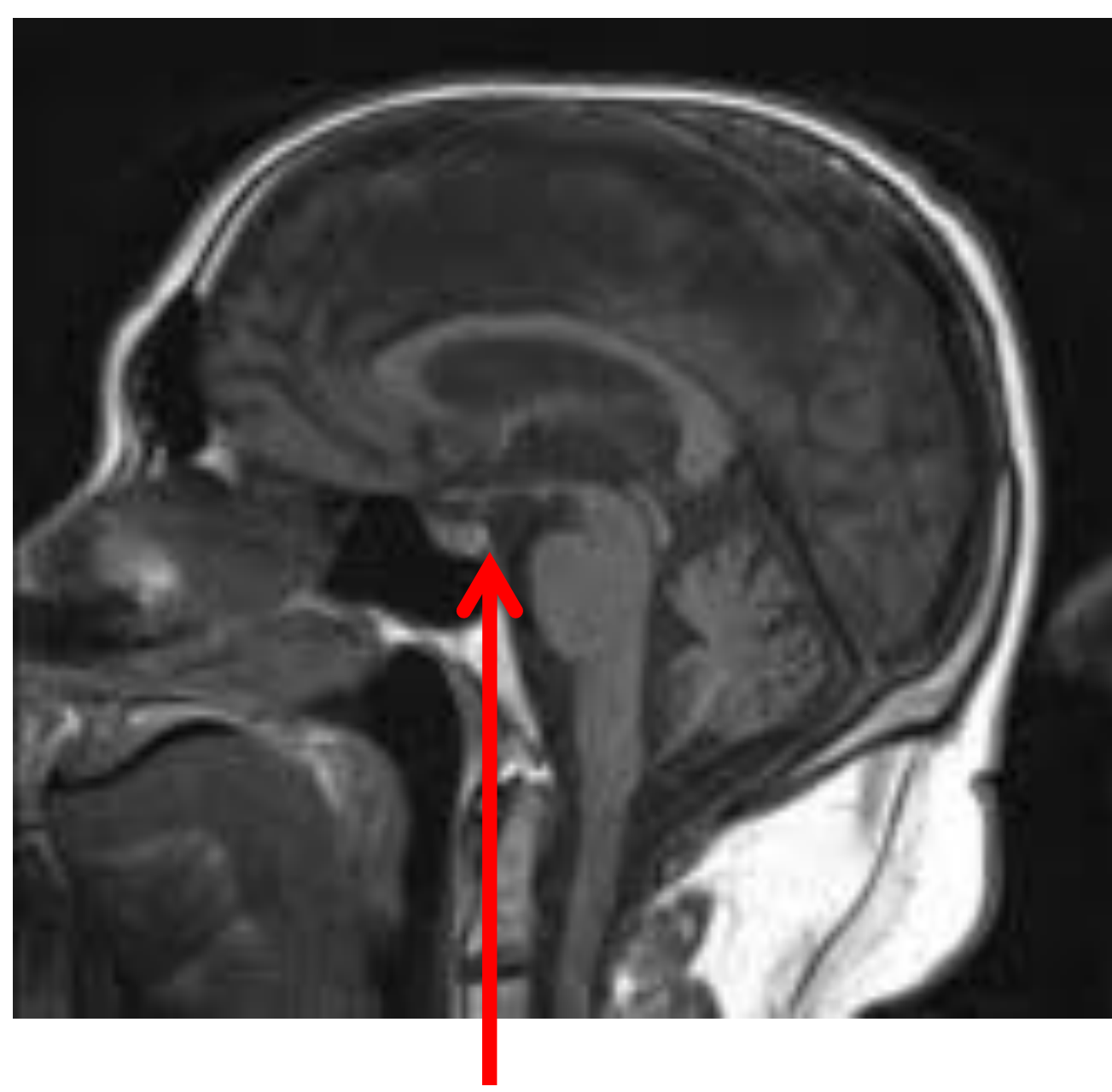

Healthy looking pituitary (patient's own)

- The Short Synacthen Test showed a low baseline cortisol of 109nmol/L which rose to $510 \mathrm{nmol} / \mathrm{L}$ after $30 \mathrm{~min}$

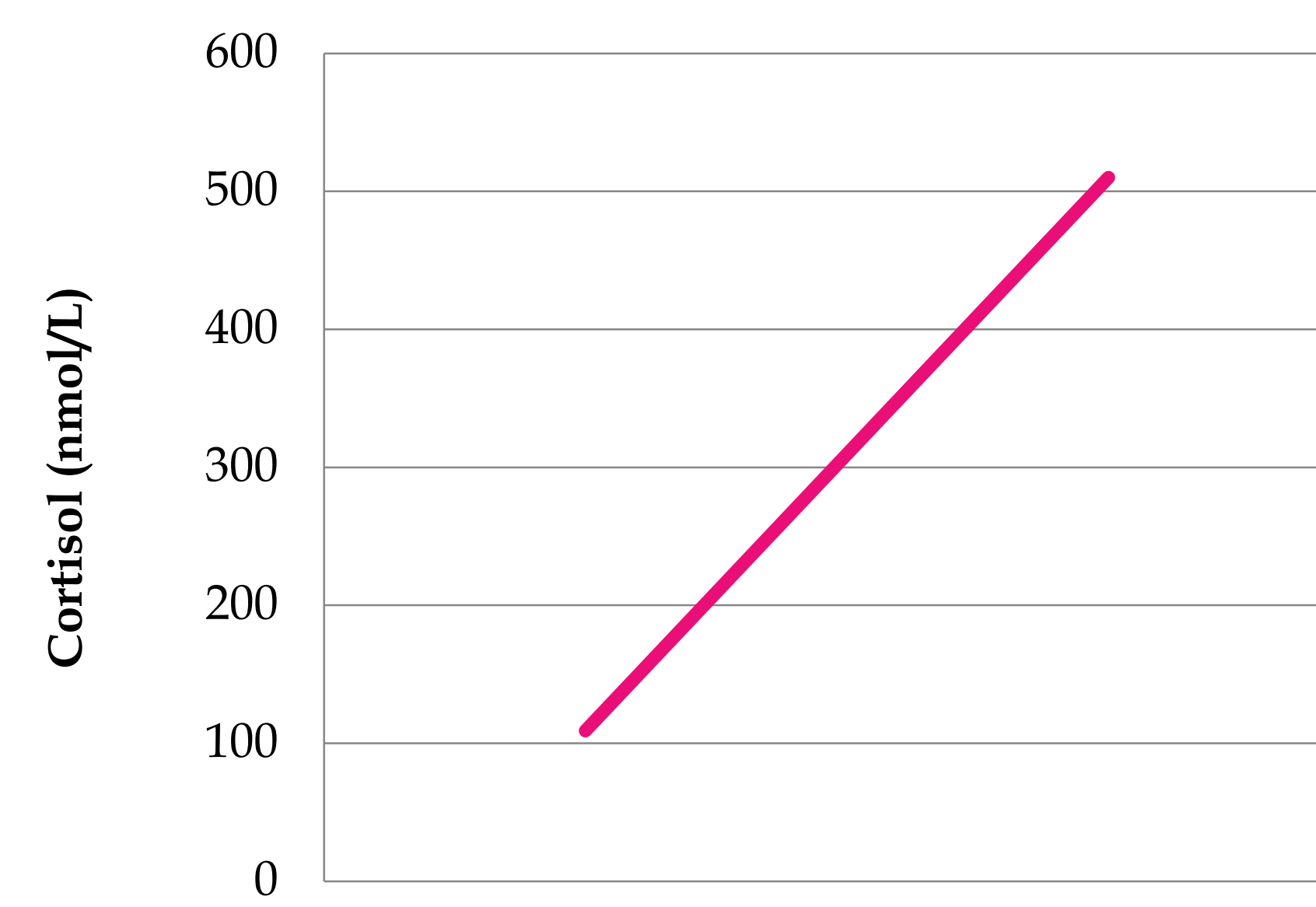

- ACTH was 14ng/1.

Lisinopril and hydrochlorothiazide were stopped and he was commenced on intravenous hydrocortisone. This led to resolution of his hypotension and hyponatraemia.

He was given a diagnosis of possible ACTH deficiency and discharged on replacement dose hydrocortisone to be followed up in the endocrine clinic.

Lisinopril and hydrochlorothiazide stopped, hydrocortisone therapy started

$$
\begin{aligned}
& \text { hydrocortisone therapy started } \quad \text { DWI restriction visible in pons } \\
& \text { pre-discharge }
\end{aligned}
$$

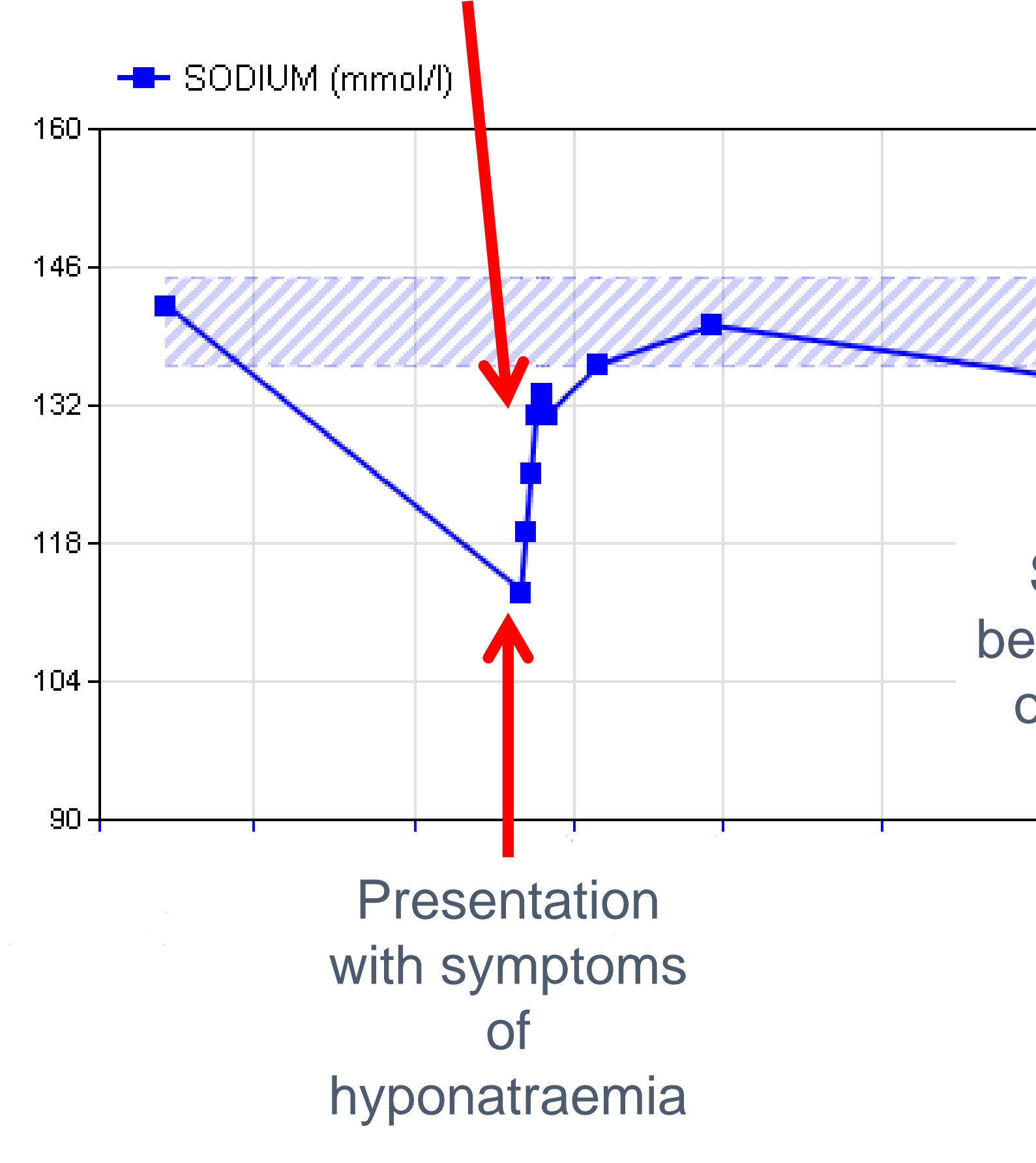

Anniversary of wife's death - alcohol consumption starts to increase again

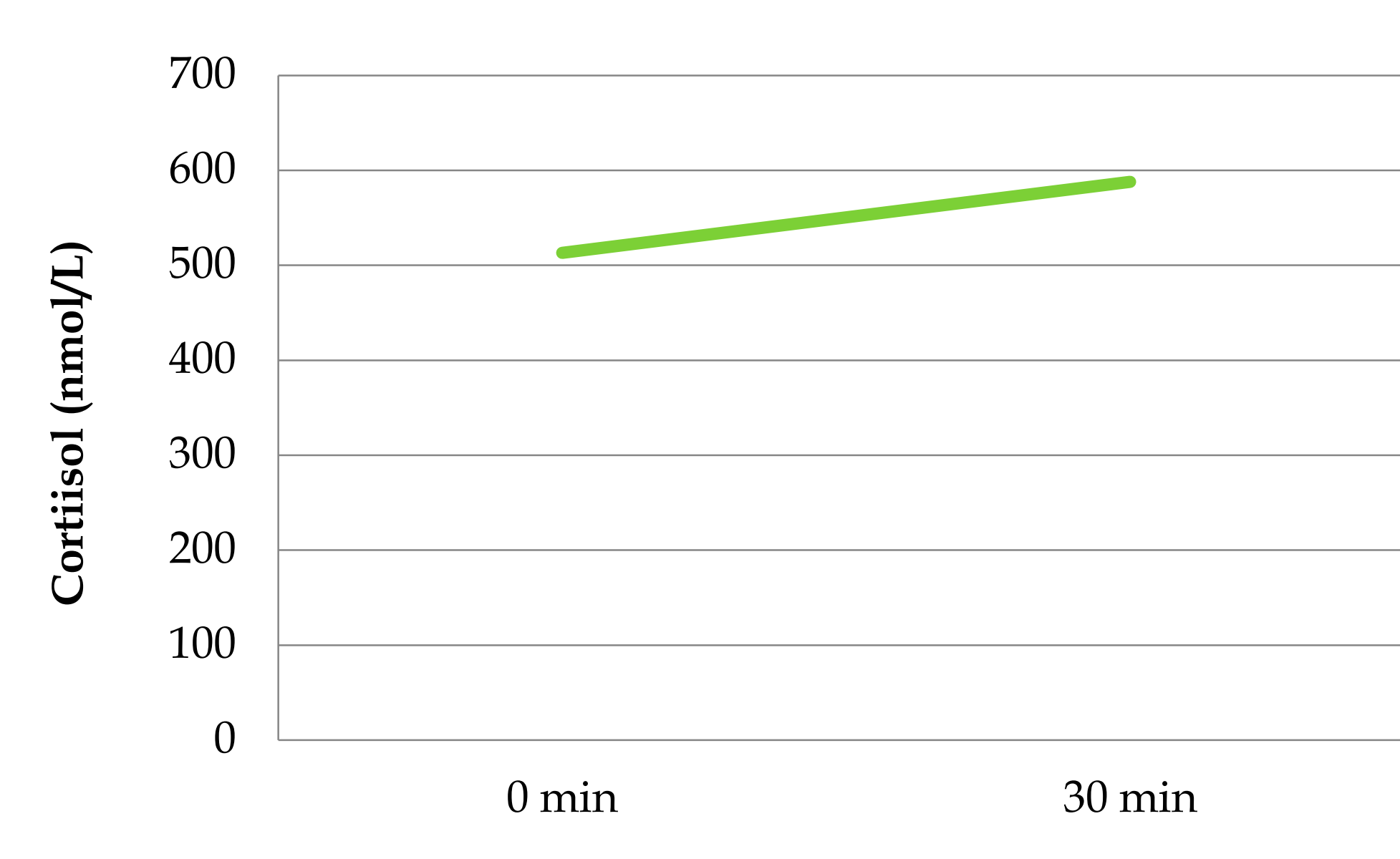

A repeat Short Synacthen Test 3 months later showed a normal baseline cortisol of $513 \mathrm{nmol} / \mathrm{L}$ rising to $588 \mathrm{nmol} / \mathrm{L}$ after $30 \mathrm{~min}$

Hydrocortisone therapy was stopped and hyponatraemia improved with changes to his antihypertensives and a reduced alcohol intake

\section{Conclusion}

The cause of this patient's initial presentation was considered to be a combination of beer

potomania is an under-recognised condition and careful fluid administration are required in the initial phase to avoid serious complication entral pontine myelinolysis

Our case demonstrates that other causes of hypotonic hyponatraemia may resulting in incorrect management.

The consequences of an incorrect diagnosis - central pontine myelinolysis

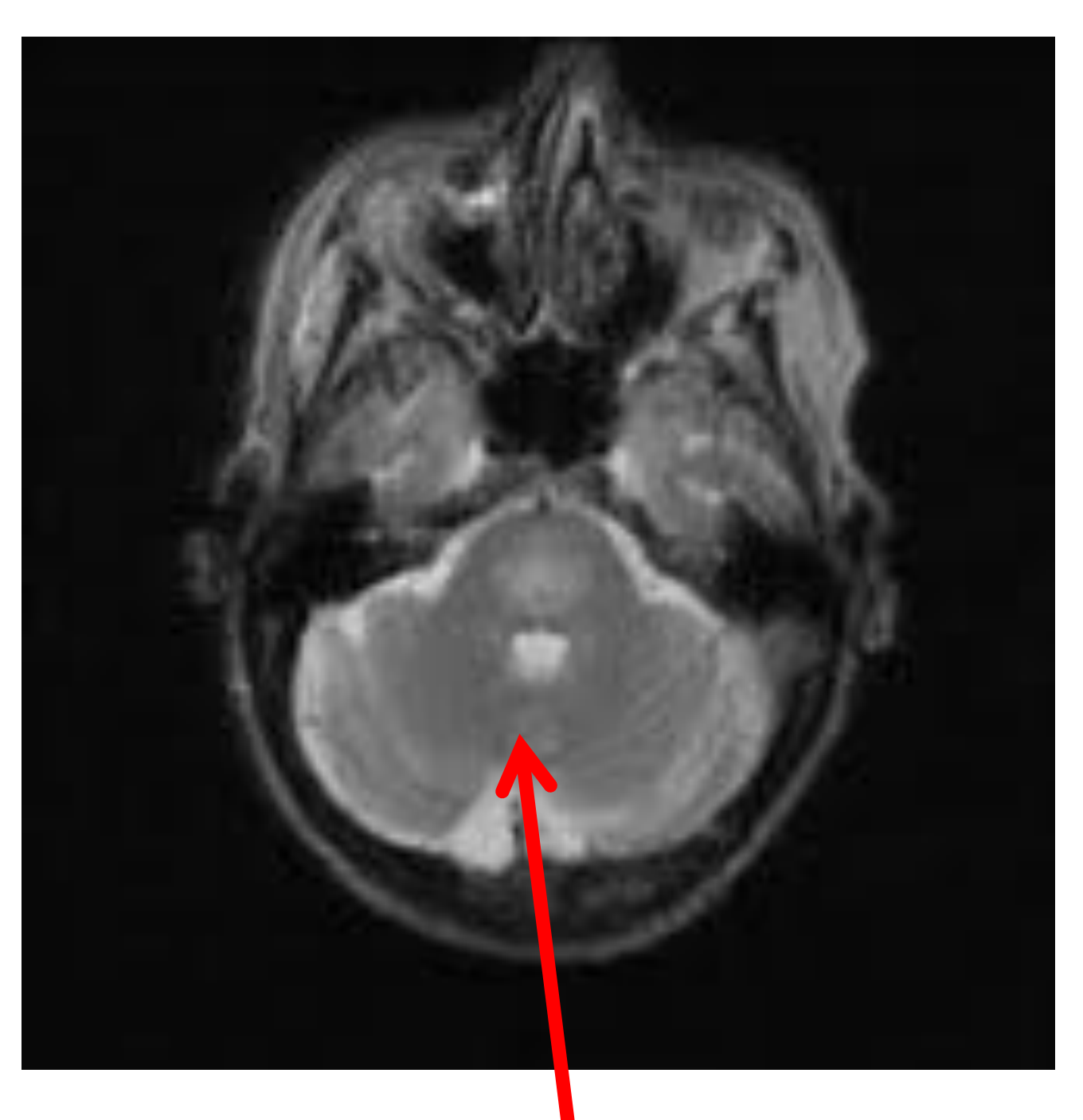$$
1
$$
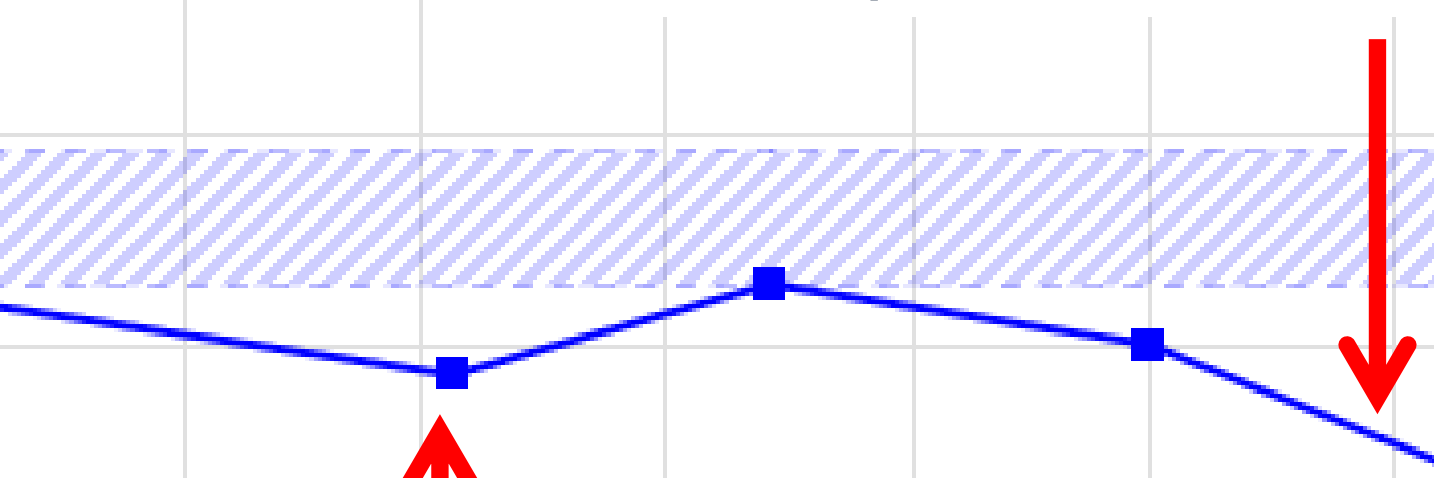

Seen in clinic - diagnosis of otomania made on review of alcohol intake - advised to stop drinking

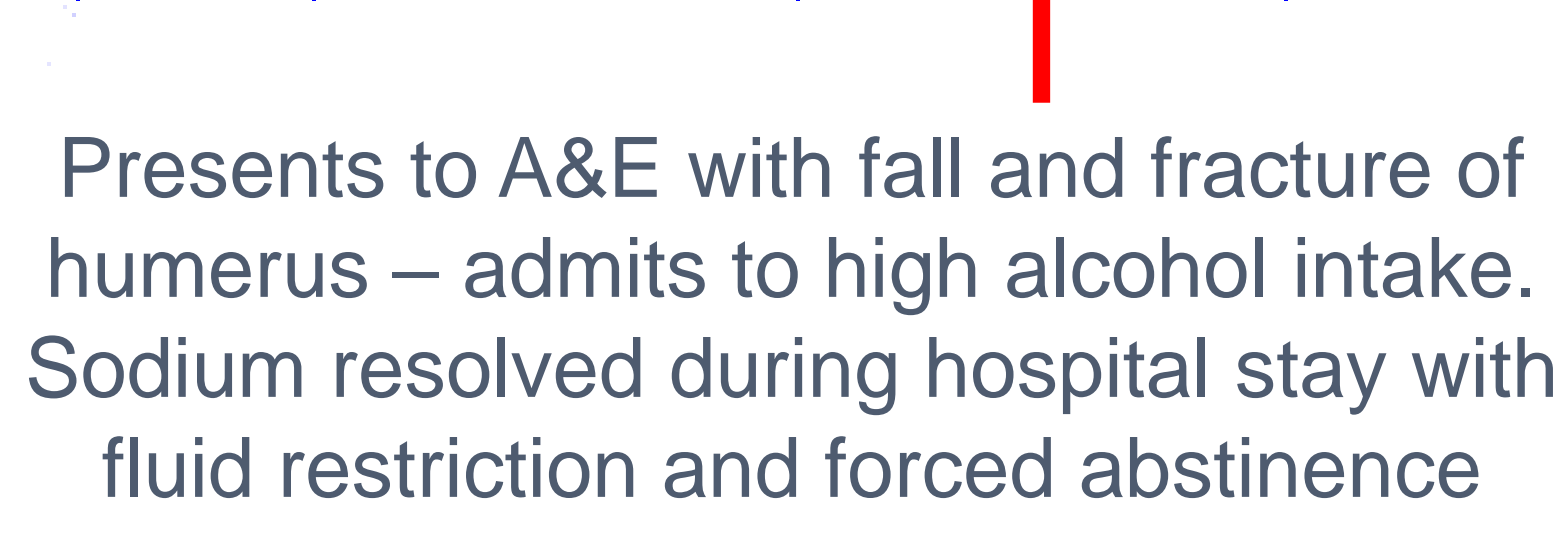
alcohol intake. fluid restriction and forced abstinence

Beer potomania was first described in $1971 .^{2}$ It is a rare disorder due to consumption of large amounts of hypotonic solution such as beer., ${ }^{1,2}$

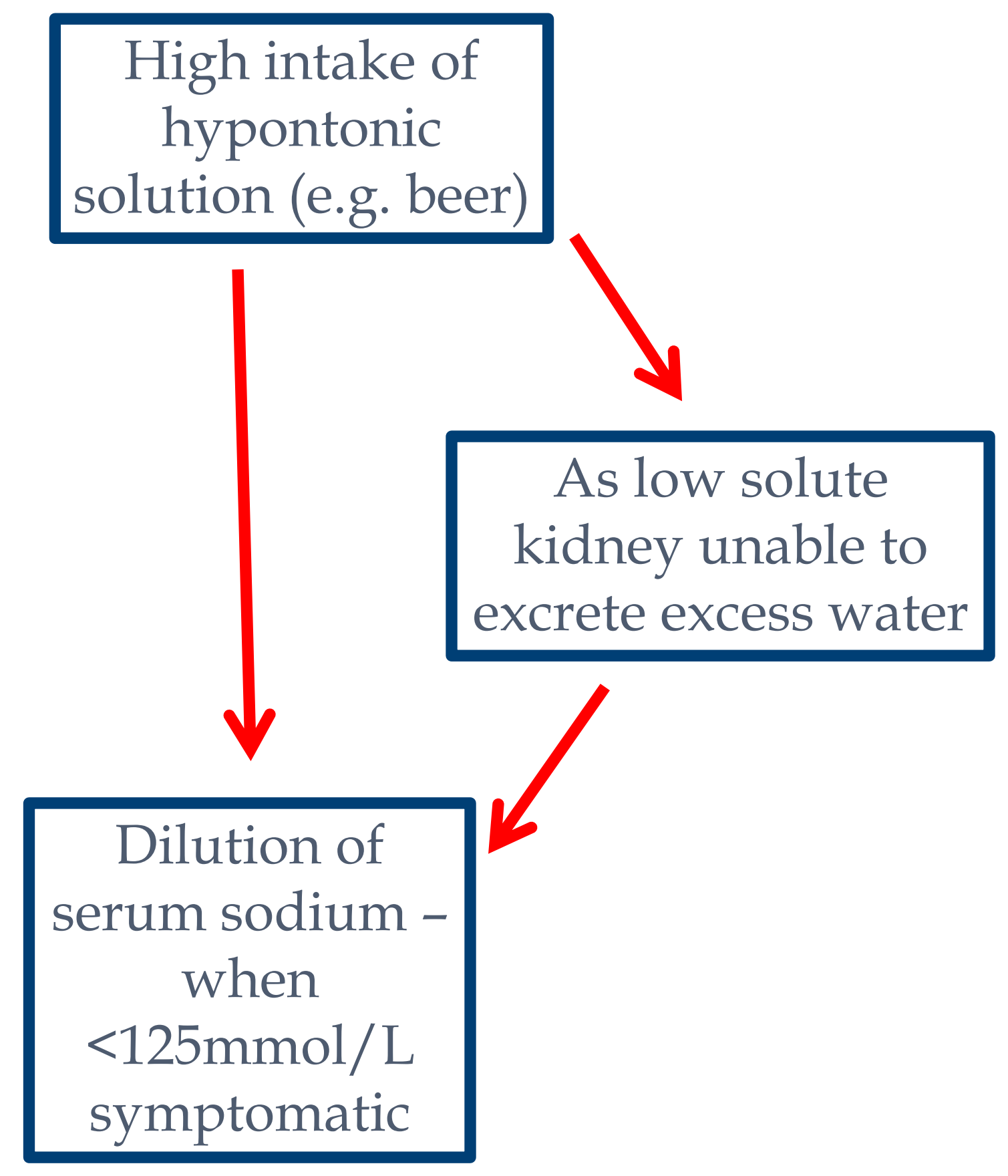

It is important to determine whether the hyponatraemia is chronic or acute. Overly aggressive treatment may cause irreversible neurological damage or death. ${ }^{1}$ There is no effective therapy after the development of central demyelination. Prevention is key. Correction of sodium should not exceed 1$2 \mathrm{mmol} / \mathrm{h}$ and $8 \mathrm{mmol} /$ day. $1,3,4$

When treating chronic hyponatraemia which is the more likely scenario with beer potomania, removal of the underlying cause and fluid restriction are the best course of treatment.

\section{Differential Diagnosis for Euvolaemic Hyponatraemia $^{4}$}

\section{Thiazide diuretics}

Hypothyroidism

Adrenal insufficiency

Syndrome of Inappropriate Anti-Diuretic Hormone secretion (cancer, CNS disorders, drugs, pulmonary disease, nausea, post-operative pain, HIV infection, Guillain-Barre syndrome, acute intermittent porphyria)

Decreased solute ingestion (beer potomania/tea and toast diet)

\section{References}

M Campbell, Hyponatraemia and central pontine myelinolysis as a result of beer potomania: a case report, Prim Care Companion J Clin Psychiatry 2010 12;4 $J$ Demanet et al., Coma due to water intoxication in beer M Biswas, J S Davies, Hyponatrae Flinicali, Hyponatraemia: a problem-solving PReddy AD Moradian, Diagnosis and ma hyponatraemia in hospitalised patients, Int $J$ Clin Pract 2009 63;10: 1494-1508

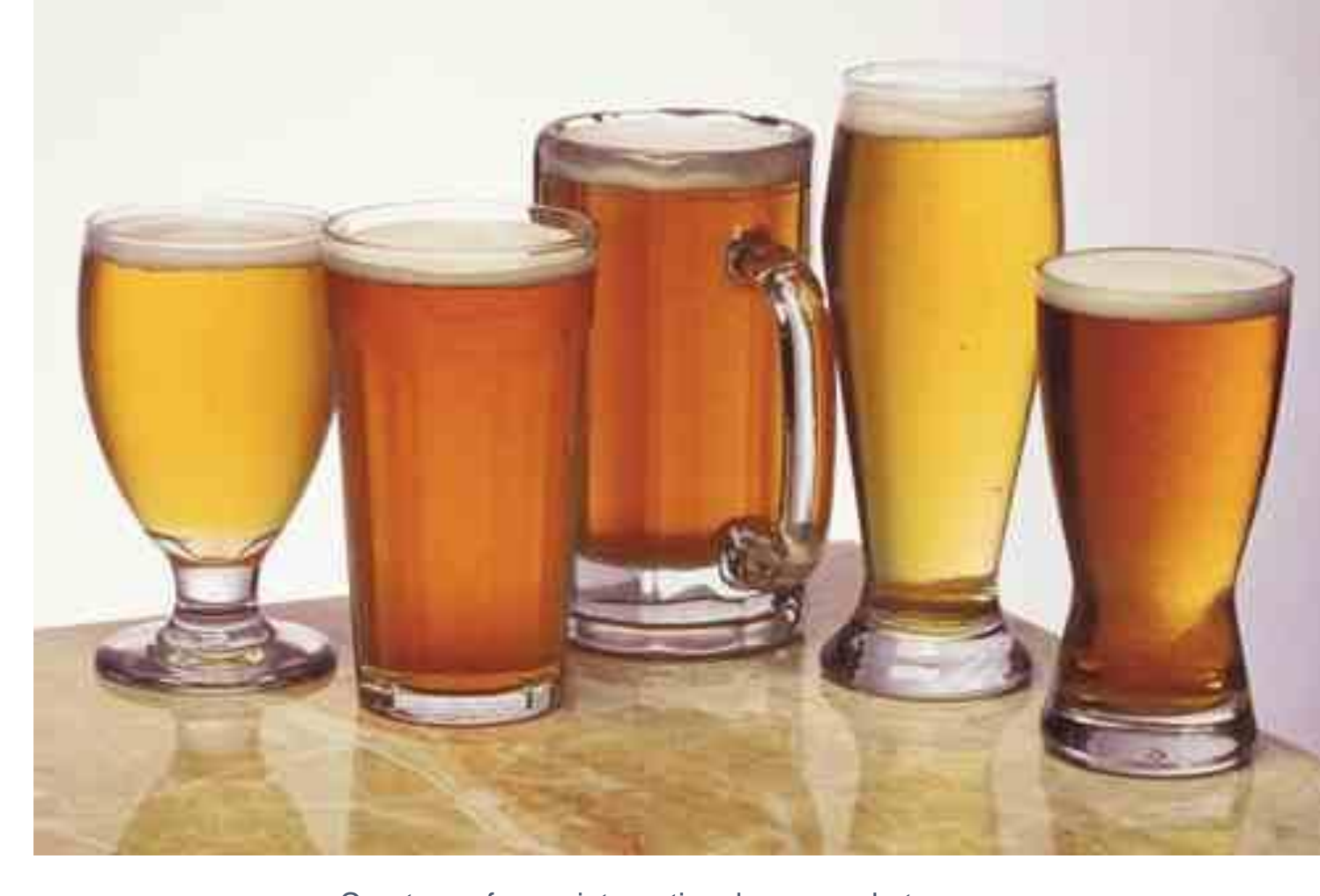

